\title{
Generalized Adiabatic Theorems: Quantum Systems Driven by Modulated Time-Varying Fields
}

\author{
Amro Dodin $\circledast^{1, *}$ and Paul Brumer $\circledast^{2, \dagger}$ \\ ${ }^{1}$ Department of Chemistry, Massachusetts Institute of Technology, Cambridge, Massachusetts 02139, USA \\ ${ }^{2}$ Chemical Physics Theory Group, Department of Chemistry and Center for Quantum Information and Quantum \\ Control, University of Toronto, Toronto, Ontario M5S 3H6, Canada
}

(Received 9 March 2021; revised 25 May 2021; accepted 4 June 2021; published 2 July 2021)

\begin{abstract}
We present generalized adiabatic theorems for closed and open quantum systems that can be applied to slow modulations of rapidly varying fields, such as oscillatory fields that occur in optical experiments and light-induced processes. The generalized adiabatic theorems show that a sufficiently slow modulation conserves the dynamical modes of time-dependent reference Hamiltonians. In the limiting case of modulations of static fields, the standard adiabatic theorems are recovered. Applying these results to periodic fields shows that they remain in Floquet states rather than in energy eigenstates. More generally, these adiabatic theorems can be applied to transformations of arbitrary time-dependent fields, by accounting for the rapidly varying part of the field through the dynamical normal modes, and treating the slow modulation adiabatically. As examples, we apply the generalized theorem to (a) predict the dynamics of a two-level system driven by a frequency-modulated resonant oscillation, a pathological situation beyond the applicability of traditional adiabatic theorems, and (b) to show that open quantum systems driven by slowly turned-on incoherent light, such as biomolecules under natural illumination conditions, can display only coherences that survive in the steady state.
\end{abstract}

DOI: 10.1103/PRXQuantum.2.030302

\section{INTRODUCTION}

Quantum systems driven by slowly varying external fields play an important role in atomic, molecular, and optical physics. The ubiquity of these processes has led to sustained interest in powerful adiabatic theorems (ATs) that characterize their dynamics [1-4]. The best-known AT states that a system initialized in an energy eigenstate will remain in that state when driven by a slowly varying field. Adiabatic processes have found far reaching utility in quantum dynamics $[5,6]$ and in the development of adiabatic quantum computing (AQC), where they are used to reliably realize quantum state transformations $[7,8]$. Moreover, adiabaticity conditions bound the allowable speed of state transformations, setting the time cost of AQC algorithms and spurring efforts to find shortcuts to adiabaticity [9-12]. However, ATs fail for oscillating fields [13-17], since these fields can induce transitions between energy

\footnotetext{
*adodin@mit.edu

†paul.brumer@utoronto.ca

Published by the American Physical Society under the terms of the Creative Commons Attribution 4.0 International license. Further distribution of this work must maintain attribution to the author(s) and the published article's title, journal citation, and DOI.
}

eigenstates, precisely the opposite of what is required in traditional adiabatic theorems. Hence traditional ATs can not, in general, be applied to light-induced processes in either isolated or open quantum systems.

Adiabatic dynamics have been successfully deployed in experimental settings through adiabatic rapid passage (ARP) [18] and stimulated Raman adiabatic passage (STIRAP) $[19,20]$ techniques. In these experiments, the adiabatic theorem provides a robust mechanism for highfidelity state preparation that is not sensitive to slight perturbations in the system properties and has been used, for example, to study molecular reaction dynamics [21] and to prepare ultracold molecules $[22,23]$. However, the domain of these techniques has been limited by the applicability of the AT, limiting the forms of driving fields and transformation times that can be realized. By relaxing key restrictions imposed by the traditional AT, the adiabatic modulation theorem (AMT) introduced below significantly broadens the range of experimental techniques, unlocking the potential for faster, more flexible state preparation and system dynamics.

Numerous scenarios do not admit traditional adiabatic approaches. Natural light-induced excitation of biological molecules (e.g., in photosynthesis or vision) provides a particularly important example of a challenge to standard ATs insofar as it involves incoherent excitation of open 
quantum systems with turn-on times that are exceedingly long compared to the time scale of molecular dynamics. For example, human blinking, the turn-on time of light in vision, occurs over $0.2 \mathrm{~s}$ as compared to the timescale of molecular isomerization induced by incident light in vision, which can, under pulsed laser excitation, occur in $50 \mathrm{fs}$ or less. Thus, challenging questions related to the creation of molecular coherences in such natural processes requires an AT adapted to oscillating incoherent fields in open quantum systems [24-27].

In this paper, we derive generalized ATs, termed adiabatic modulation theorems, for open and closed quantum systems that apply to oscillatory and other rapidly varying fields, extending adiabaticity conditions to optically driven processes and providing faster pathways to adiabaticity, with the potential to accelerate AQC. Moreover, we show that the adiabatic transformation time is limited not by the energy gaps in the systems but rather by the frequency differences of the instantaneous normal modes, which are often easier to manipulate. These results exploit the fact that many complicated fields of interest are time-dependent modulations (e.g., frequency- and amplitude-modulated oscillations) of simpler fields that have well-understood dynamics.

The AMTs derived below show that a system subjected to a sufficiently slow modulation of a time-dependent field conserves the dynamical modes of time-dependent reference Hamiltonians. These theorems contain traditional ATs in the limit of modulations of static fields, and generalize easily to modulations of periodic fields, which are shown to preserve Floquet states rather than energy eigenstates. Moreover, we show that the adiabatic transformation time is limited not by the energy gaps in the system but rather by the frequency differences of the instantaneous normal modes, that are often easier to manipulate. Remarkably, these constructions allow for the design of experimental techniques that go beyond the preparation of time-independent states and allow for the preparation of specific dynamics using the well-developed intuition of adiabatic theory.

The AMT theorems are widely applicable and two significant examples are provided below. In the isolated system example, we show how the theorem allows for control of dynamics on the entire Bloch sphere in a two-level qubit system. In applications to open systems, the theorem resolves a long-standing controversy regarding the role of light-induced coherent oscillations in biophysical processes. The generality of the theorems ensure applications to a wide variety of alternative systems.

This paper is organized as follows: Sec. II proves the adiabatic modulation theorems for both isolated and open systems. Sample applications, designed to demonstrate the theorems and their applications are provided in Sec. III. Section IV provides a conclusion.

\section{THE ADIABATIC MODULATION THEOREMS}

\section{A. Isolated systems}

Consider a family of time-dependent reference Hamiltonians $\hat{H}_{0}(t ; \lambda)$ indexed by the parameter $\lambda$ [e.g., $\lambda$ may be an amplitude $\hat{H}_{0}(t ; \lambda)=\lambda \hat{h}_{0}(t)$ or frequency $\hat{H}_{0}(t ; \lambda)=$ $\exp (-i \lambda t) \hat{h}_{0}+$ h.c.]. For each value of $\lambda$, setting $\hbar=1$, we define the instantaneous normal modes as

$$
\left|\psi_{n}(t ; \lambda)\right\rangle \equiv e^{-i \theta_{n}(t ; \lambda)}|n(t ; \lambda)\rangle,
$$

where $\theta_{n}(t ; \lambda) \equiv \int_{0}^{t} d s \epsilon_{n}(s ; \lambda)$ is the dynamical phase, $\epsilon_{n}(t ; \lambda)$ is an instantaneous quasienergy and the $|n(t ; \lambda)\rangle$ is the instantaneous normal mode of $\hat{H}_{0}(t ; \lambda)$.

These normal modes and corresponding quasienergies are found by solving the eigenproblem

$$
\hat{H}_{F}(t ; \lambda)|n(t ; \lambda)\rangle=\epsilon_{n}(t ; \lambda)|n(t ; \lambda)\rangle,
$$

where $\hat{H}_{F}(t ; \lambda) \equiv \hat{H}_{0}(t ; \lambda)-i \partial / \partial t$. That is, the normal modes are particular solutions to the time-dependent Schrödinger equation (TDSE),

$$
i \frac{\partial}{\partial t}|\Psi(t)\rangle=\hat{H}(t)|\Psi(t)\rangle,
$$

and are well understood for many driving fields. For example, for time-independent Hamiltonians, the normal modes of static Hamiltonians are energy eigenstates, while for periodic Hamiltonians they are Floquet states.

A modulation of a time-dependent Hamiltonian, $\hat{H}_{0}(t ; \lambda)$, is a transformation that varies $\lambda \rightarrow \lambda_{t}$ over a time interval $[0, \tau]$ through a modulation protocol $\Lambda \equiv\left\{\lambda_{t} \mid t \in\right.$ $[0, \tau]\}$. The resultant modulated field $\hat{H}_{\Lambda}(t) \equiv \hat{H}_{0}\left(t ; \lambda_{t}\right)$ sweeps through the Hamiltonian family defined above [e.g., a field with a time-dependent amplitude for $\hat{H}_{0}=$ $\left.\lambda h_{0}(t)\right]$. Note, as an aside, that this is an operator generalization of modulations in signal processing and encoding theory [28]. Generally, the modulated Hamiltonian can be significantly more complicated than $H_{0}$ since the modulation function may be highly nonlinear and the modulation parameter can vary nontrivially with time.

The dynamics induced by $\hat{\widetilde{H}}_{\Lambda}(t)$ can now be expressed in terms of instantaneous normal modes of $\hat{H}_{0}$, Eq. (1), to give

$$
|\Psi(t)\rangle \equiv \sum_{n} c_{n}(t) e^{-i \theta_{n}(t ; \Lambda)}\left|n\left(t ; \lambda_{t}\right)\right\rangle,
$$

where $\theta_{n}(t ; \Lambda) \equiv \int_{0}^{t} d s \epsilon_{n}\left(s ; \lambda_{s}\right) / \hbar$. The instantaneous state of the system can always be expressed in this form since $\hat{H}_{F}\left(t ; \lambda_{t}\right)$ is always Hermitian and therefore has a basis of eigenstates $\left\{\left|n\left(t ; \Lambda_{t}\right)\right\rangle\right\}$ that can be used to expand $|\Psi(t)\rangle$ with coefficient $a_{n}(t) \equiv\left\langle n\left(t ; \lambda_{t}\right) \mid \Psi(t)\right\rangle=$ $c_{n}(t) \exp \left[-i \theta_{n}(t)\right][29]$. 
This approach mirrors that used to derive traditional ATs, but replacing the eigenstates with normal modes and energies with quasienergies. We therefore proceed through a similar path to derive adiabaticity conditions under which a system initialized in a normal mode of $\hat{H}_{0}\left(0 ; \lambda_{0}\right)$ will remain in that normal mode at all times. Mathematically, this occurs when $c_{n}(t)$ are decoupled in the TDSE, Eq. (3). Substituting Eq. (4) into Eq. (3) we find

$$
\sum_{n}\left(\dot{c}_{n}(t)\left|n\left(t ; \lambda_{t}\right)\right\rangle+c_{n}(t) \dot{\lambda}_{t} \frac{\partial}{\partial \lambda_{t}}\left|n\left(t ; \lambda_{t}\right)\right\rangle\right) e^{-i \theta_{n}(t ; \Lambda)}=0,
$$

where we use the identity $d \theta_{n}(t ; \Lambda) / d t=\epsilon_{n}\left(t ; \lambda_{t}\right)$ and Eq. (2) to cancel terms proportional to $\epsilon_{n}\left(t ; \lambda_{t}\right)$. Projecting onto an $\left|m\left(t ; \lambda_{t}\right)\right\rangle$, yields equations of motion for the coefficients,

$$
\begin{aligned}
\dot{c}_{m}(t)= & -c_{m}(t) \dot{\lambda}_{t}\left\langle m\left|\frac{\partial}{\partial \lambda_{t}}\right| n\right\rangle \\
& +\sum_{n \neq m} c_{n}(t) \frac{\dot{\lambda}_{t} e^{-i \theta_{n m}}\langle m|\partial \hat{\widetilde{H}} / \partial \lambda| n\rangle}{\epsilon_{m}-\epsilon_{n}},
\end{aligned}
$$

where we suppress the $t$ and $\lambda_{t}$ dependence for brevity, and $\theta_{n m} \equiv \theta_{n}-\theta_{m}$. To obtain Eq. (6), Eq. (2) is differentiated with respect to $\lambda_{t}$ to obtain $\left\langle m\left|\partial / \partial \lambda_{t}\right| n\right\rangle=$ $\left\langle m\left|\partial \hat{\widetilde{H}} / \partial \lambda_{t}\right| n\right\rangle /\left(\epsilon_{n}-\epsilon_{m}\right)$.

Equation (6) takes the same form as the original adiabatic theorem with cross-coupling between normal modes scaled by a rapidly oscillating term [3]. As a result normal modes evolve independently of one another when the following condition is satisfied:

$$
\max _{0 \leq t \leq \tau}\left|\frac{\dot{\lambda}_{t}\left\langle m\left|\partial \hat{H}\left(t ; \lambda_{t}\right) / \partial \lambda_{t}\right| n\right\rangle}{\epsilon_{n m}\left(t ; \lambda_{t}\right)}\right| \ll \min _{0 \leq t \leq \tau}\left|\epsilon_{n m}\left(t ; \lambda_{t}\right)\right|,
$$

where $\epsilon_{n m}\left(t ; \lambda_{t}\right) \equiv \epsilon_{n}\left(t ; \lambda_{t}\right)-\epsilon_{m}\left(t ; \lambda_{t}\right)$. Consequently, if $\lambda_{t}$ changes much more slowly than the difference in dynamical mode frequencies, the slow modulation will not crosscouple the normal modes of the modulated Hamiltonian.

Equation (7) has several important features. First, if $\hat{H}_{0}(t ; \lambda)$ are $t$ independent, then their normal modes are energy eigenstates and all Hamiltonian time dependence is contained in the modulation. In this case, the AMT reduces to the traditional AT, simply relabeling the time variable as $\lambda$. The AMT then extends previous ATs by recognizing them as statements about how time-dependent transformations of Hamiltonian fields impact dynamical modes of the TDSE. These coincide with eigenstates for static Hamiltonians but the same insight can be applied to any field with well-understood dynamics. For example, if $\hat{H}_{0}(t ; \lambda)$ are periodic, Eq. (7) states that slow modulations do not couple Floquet states. One key distinction of the
AMT is that its speed limits are set by the differences in normal-mode frequencies, which are often easier to manipulate than energy gaps between eigenstates, allowing for faster adiabatic transformations. A numerical example is discussed in Sec. III A1.

\section{B. Open systems}

Consider now an adiabatic theorem for open quantum systems. To prove this theorem we take an approach inspired by Ref. [3]. The dynamics of an open quantum system are governed by the generalized Liouville-von Neumann (LvN) equation

$$
\left.\left.\frac{d}{d t}|\rho\rangle\right\rangle=\hat{\hat{\mathcal{L}}}(t)|\rho\rangle\right\rangle
$$

in the time-convolutionless form [30-32]. The Liouville super operator $\hat{\hat{L}}(t)$ in Eq. (8) describes both unitary dynamics and nonunitary relaxation processes such as dephasing and dissipation. For example, in the Markovian limit, Eq. (8) can be written in the Lindblad form [30], consisting of a unitary component $-i / \hbar[\hat{H}, \hat{\rho}]$ and a sum of nonunitary Lindblad dissipators. The double-ket notation $|\cdot\rangle\rangle$ indicates an operator Liouville space with a trace inner product $\langle\langle A \mid B\rangle\rangle \equiv \operatorname{Tr}\left\{\hat{A}^{\dagger} \hat{B}\right\}$. By analogy with the isolated system, we define modulations by starting with a family of time-dependent Liouvillians $\hat{\hat{\mathcal{L}}}_{0}(t ; \lambda)$ and allow the modulation parameter to vary over time to give $\hat{\hat{\mathcal{L}}}_{\Lambda}(t) \equiv$ $\hat{\hat{\mathcal{L}}}_{0}\left(t ; \lambda_{t}\right)$. A modified Liouvillian can then be defined by $\hat{\hat{\mathcal{L}}}_{F}(t ; \lambda) \equiv \hat{\hat{\mathcal{L}}}(t ; \lambda)-\partial / \partial t$.

The LvN equation appears similar to the TDSE [Eq. (3)], suggesting that we may be able to apply the same analysis, but replacing Hamiltonians with Liouvillians. However, the Liouvillian and its corresponding modified operator are completely positive but not necessarily Hermitian [31] and therefore require more care in defining their normal modes. A given $M \times M$ modified Liouvillian, $\hat{\hat{\mathcal{L}}}_{F}$, has an incomplete set of $N \leq M$ left and right eigenvectors $\left\langle\left\langle\mathcal{Q}_{\alpha}(t)\right|\right.$ and $\left.\left|\mathcal{P}_{\alpha}(t)\right\rangle\right\rangle$. Associated with each eigenvector is a Jordan block comprised of $n_{\alpha}$ generalized eigenvectors that combine to give an orthonormal basis of Liouville space defined by the generalized eigenproblem

$$
\begin{aligned}
& \left\langle\left\langle\mathcal{Q}_{\alpha}^{(j)}\right| \hat{\hat{\mathcal{L}}}_{F}=\left\langle\left\langle\mathcal{Q}_{\alpha}^{(j)}\right| \chi_{\alpha}+\left\langle\left\langle\mathcal{Q}_{\alpha}^{(j+1)}\right|,\right.\right.\right. \\
& \left.\left.\left.\hat{\hat{\mathcal{L}}}_{F}\left|\mathcal{P}_{\alpha}^{(j)}\right\rangle\right\rangle=\chi_{\alpha}\left|\mathcal{P}_{\alpha}^{(j)}\right\rangle\right\rangle+\left|\mathcal{P}_{\alpha}^{(j-1)}\right\rangle\right\rangle,
\end{aligned}
$$

where $\left\langle\left\langle\mathcal{Q}_{\alpha}^{\left(n_{\alpha}-1\right)}\right| \equiv\left\langle\left\langle\mathcal{Q}_{\alpha}\right|,\left\langle\left\langle\mathcal{Q}_{\alpha}^{\left(n_{\alpha}\right)}\left|\equiv\left\langle\left\langle 0|,| \mathcal{P}_{\alpha}^{(0)}\right\rangle\right\rangle \equiv\right| \mathcal{P}_{\alpha}\right\rangle\right\rangle\right.\right.$, and $\left.\left.\left|\mathcal{P}_{\alpha}^{(-1)}\right\rangle\right\rangle \equiv|0\rangle\right\rangle$, where $\left.|0\rangle\right\rangle$ is the zero operator. Dynamically, the LvN equation does not cross-couple the Jordan blocks to one another, but can lead to crosscoupling within states of the same Jordan block, thereby 
defining decoupled dynamical subspace. (For a concise summary of Jordan canonical form in the context of quantum adiabatic theorems, see Ref. [3].)

Motivated by the isolated systems' derivation, we consider the modified Liouvillian and aim to expand the dynamics of the open system in terms of its instantaneous generalized eigenstates. These are given by the (right) generalized equation

$$
\left.\left.\left.\hat{\hat{\mathcal{L}}}_{F}\left(t ; \lambda_{t}\right)\left|\mathcal{P}_{\alpha}^{(j)}\right\rangle\right\rangle=\chi_{\alpha}\left|\mathcal{P}_{\alpha}^{(j)}\right\rangle\right\rangle+\left|\mathcal{P}_{\alpha}^{(j-1)}\right\rangle\right\rangle .
$$

The left generalized eigenstates can be similarly defined as the right eigenstates of $\hat{\hat{\mathcal{L}}}_{F}^{\dagger}$.

Allowing the modulation parameter to change with time, the instantaneous state of the system can be expanded as a superposition over the generalized eigenstates in the form

$$
\left.|\rho(t)\rangle\rangle \equiv \sum_{\beta=1}^{N} \sum_{j=0}^{n_{\beta}-1} r_{\beta}^{(j)}(t)\left|\mathcal{P}_{\beta}^{(j)}\left(t ; \lambda_{t}\right)\right\rangle\right\rangle
$$

where $r_{\beta}^{(j)}(t) \equiv\left\langle\left\langle\mathcal{Q}_{\beta}^{(j)}\left(t ; \lambda_{t}\right) \mid \rho(t)\right\rangle\right\rangle$ is a complex-valued expansion coefficient. Similarly, to Eq. (4), any density operator $|\rho\rangle\rangle$ can be expressed in this form since the generalized eigenbasis that generates Jordan canonical form is complete and orthonormal.

Substituting the trial form of Eq. (11) into the timeconvolutionless Liouville-von Neumann equation [Eq. (8)] and projecting onto the left generalized eigenstate $\left\langle\left\langle\mathcal{Q}_{\alpha}^{(i)}\right|\right.$, gives the following equation of motion for the expansion coefficients:

$$
\dot{r}_{\alpha}^{(i)}=\chi_{\alpha} r_{\alpha}^{(i)}+r_{\alpha}^{(i+1)}-\sum_{\beta=1}^{N} \sum_{j=0}^{n_{\beta}-1} \dot{\lambda}_{t} r_{\beta}^{(j)}\left\langle\left\langle\mathcal{Q}_{\alpha}^{(i)}\left|\frac{\partial}{\partial \lambda_{t}}\right| \mathcal{P}_{\beta}^{(j)}\right\rangle\right\rangle
$$

where we use the Floquet Eq. (10) and the orthonormality condition of generalized eigenstates. By convention, we take $r_{\alpha}^{\left(n_{\alpha}\right)}=0$.

Differentiating Eq. (10) for some right eigenstate $\left.\left|\mathcal{P}_{\beta}^{(j)}\right\rangle\right\rangle$ with respect to $\lambda_{t}$ at fixed $t$ and projecting onto left eigenstate $\left\langle\left\langle\mathcal{Q}_{\alpha}^{(i)}\right|\right.$ with $\alpha \neq \beta$ gives

$$
\begin{aligned}
\left\langle\left\langle\mathcal{Q}_{\alpha}^{(i)}\right|\right. & \left.\frac{\partial \hat{\hat{\mathcal{L}}}}{\partial \lambda_{t}}\left|\mathcal{P}_{\beta}^{(j)}\right\rangle\right\rangle+\left\langle\left\langle\mathcal{Q}_{\alpha}^{(i)}\left|\hat{\hat{\mathcal{L}}} \frac{\partial}{\partial \lambda_{t}}\right| \mathcal{P}_{\beta}^{(j)}\right\rangle\right\rangle \\
= & \frac{\partial \chi_{\beta}}{\partial \lambda_{t}}\left\langle\left\langle\mathcal{Q}_{\alpha}^{(i)} \mid \mathcal{P}_{\beta}^{(j)}\right\rangle\right\rangle+\chi_{\beta}\left\langle\left\langle\mathcal{Q}_{\alpha}^{(i)} \mid \dot{\mathcal{P}}_{\beta}^{(j)}\right\rangle\right\rangle \\
& +\left\langle\left\langle\mathcal{Q}_{\alpha}^{(i)} \mid \dot{\mathcal{P}}_{\beta}^{(j-1)}\right\rangle\right\rangle .
\end{aligned}
$$

This expression can be simplified by first noting that for $\alpha \neq \beta$ orthonormality of the basis eliminates the $\left\langle\left\langle\mathcal{Q}_{\alpha}^{(i)} \mid \mathcal{P}_{\beta}^{(j)}\right\rangle\right\rangle$ term on the right-hand side of Eq. (13). Equation (9a) can then be used to expand the second term, $\left\langle\left\langle\mathcal{Q}_{\alpha}^{(i)}\left|\hat{\hat{\mathcal{L}}}\left(\partial / \partial \lambda_{t}\right)\right| \mathcal{P}_{\beta}^{(j)}\right\rangle\right\rangle$ yielding a recursive expression for the projected change of the normal modes.

$$
\left\langle\left\langle\mathcal{Q}_{\alpha}^{(i)}\left|\frac{\partial}{\partial \lambda_{t}}\right| \mathcal{P}_{\beta}^{(j)}\right\rangle\right\rangle=\frac{1}{\chi_{\beta \alpha}}\left(\left\langle\left\langle\mathcal{Q}_{\alpha}^{(i)}\left|\frac{\partial \hat{\mathcal{L}}}{\partial \lambda_{t}}\right| \mathcal{P}_{\beta}^{(j)}\right\rangle\right\rangle+\left\langle\left\langle\mathcal{Q}_{\alpha}^{(i+1)}\left|\frac{\partial}{\partial \lambda_{t}}\right| \mathcal{P}_{\beta}^{(j)}\right\rangle\right\rangle-\left\langle\left\langle\mathcal{Q}_{\alpha}^{(i)}\left|\frac{\partial}{\partial \lambda_{t}}\right| \mathcal{P}_{\beta}^{(j-1)}\right\rangle\right\rangle\right),
$$

where $\chi_{\beta \alpha} \equiv \chi_{\beta}-\chi_{\alpha}$. Iterating recursively through Eq. (14), the change in the normal modes can be related to the change in the modulated Liouvillian by

$$
\begin{aligned}
& \left\langle\left\langle\mathcal{Q}_{\alpha}^{(i)}\left|\frac{\partial}{\partial \lambda}\right| \mathcal{P}_{\beta}^{(j)}\right\rangle\right\rangle \\
& =\sum_{p=1}^{n_{\alpha}-i j-S_{0}} \sum_{k_{1}=0} \cdots \sum_{k_{p}=0}^{j-S_{p}} \frac{\left\langle\left\langle\mathcal{Q}_{\alpha}^{(i+p-1)}|\partial \hat{\mathcal{L}} / \partial \lambda| \mathcal{P}_{\beta}^{\left(j-S_{p}\right)}\right\rangle\right\rangle}{(-1)^{S_{p}} \chi_{\beta \alpha}^{p+S_{p}}},
\end{aligned}
$$

where $S_{q} \equiv \sum_{s=1}^{q} k_{s}$, and $k_{s}$ are the summation indexes over states in the Jordan block.

Finally, by substituting Eq. (15) into Eq. (12) and considering only the terms that couple nondegenerate Jordan blocks $\alpha \neq \beta$, we obtain an adiabaticity condition for slowly modulated open quantum systems:

$$
\begin{aligned}
& \max _{0 \leq t \leq T} \mid \sum_{p=1}^{n_{\alpha}-i j-S_{0}} \sum_{k_{1}=0}^{j-S_{p}} \cdots \sum_{k_{p}=0}^{\left\langle\left\langle\mathcal{Q}_{\alpha}^{(i+p-1)}|\partial \hat{\hat{\mathcal{L}}} / \partial \lambda| \mathcal{P}_{\beta}^{\left(j-S_{p}\right)}\right\rangle\right\rangle} \\
& \quad \ll \min _{0 \leq t \leq T}\left|\dot{\lambda}_{t}^{-1}\right| .
\end{aligned}
$$

This expression is admittedly unwieldy but provides a completely general criteria for open system adiabaticity.

While the Jordan form treatment is required for open systems dynamics in full generality, in many cases the Liouvillian can be diagonalized, i.e., making all Jordan blocks one dimensional. In this case, the recursive iteration in Eq. (14) and subsequent summations in Eqs. (15) and (16) are not required and a treatment analogous to the 
closed systems case can be used. The resulting expression then takes the same form as Eq. (7) with the slight modification that the Liouvillian eigenvalues $\chi_{\alpha}$ are generally complex.

A number of simpler bounds, albeit less tight than Eq. (16), can be obtained for the adiabaticity condition by extending the approach discussed in Ref. [3] that treated constant Liouvillian. The simplest of these results states that if $\dot{\lambda}_{t} \rightarrow 0$, e.g., in Eq. (12), then the open quantum system undergoes adiabatic dynamics. Moreover, similarly to the closed system adiabatic theorem, Eq. (16) can be used to derive the earlier nonmodulated adiabatic theorem of Ref. [3] by assuming a constant $\hat{\mathcal{L}}_{0}(t)$.

A significant example of the open system AMT is provided in Sec. III B where the theorem is applied to the slowly turned-on incoherent (e.g., solar radiation) excitation of molecular systems.

\section{COMPUTATIONAL EXAMPLES}

\section{A. Isolated systems \\ 1. Rabi-type system}

In this section, we consider a two-level system (TLS) driven by a frequency-modulated oscillatory field. We begin by defining an extension of the Rabi model, the family of Hamiltonians,

$$
\hat{H}_{0}(t ; \omega)=\left(\begin{array}{cc}
-\Delta_{0} & V e^{i \omega t} \\
V^{*} e^{-i \omega t} & \Delta_{0}
\end{array}\right)
$$

that characterize driving by a field with frequency $\omega$. Here $\Delta_{0}$ is the energy difference between the states $|0\rangle$ and $|1\rangle$, the coupling coefficient is given by $V=\mathbf{E} \cdot \boldsymbol{\mu}_{01}, \mathbf{E}$ is the electric field vector driving the system, $\boldsymbol{\mu}_{\mathbf{0 1}}$ is the transition dipole moment between the two states and we set $\hbar=1$. This family of Hamiltonians comprises the standard Rabi model with well-understood dynamics for all values of $\omega$.

It is useful to briefly review the dynamics induced by Eq. (17) from the perspective of Floquet's theorem to highlight the normal modes that play an important role in the generalized adiabatic theorem. Since $\hat{H}_{0}(t)$ is periodic with period $T=2 \pi / \omega$, its dynamical normal modes are two $T$-periodic Floquet states, $| \pm(t ; \omega)\rangle$ with timeindependent quasienergies $\epsilon_{ \pm}$. These states are eigenstates of the Floquet Hamiltonian, satisfying

$$
\begin{aligned}
& \hat{H}_{F}(t ; \omega)| \pm(t ; \omega)\rangle=\epsilon_{ \pm}| \pm(t ; \omega)\rangle, \\
& \hat{H}_{F}(t ; \omega) \equiv \hat{H}_{0}(t ; \omega)-i \frac{\partial}{\partial t} .
\end{aligned}
$$

For the Hamiltonian in Eq. (17), these Floquet states and quasienergies are given by

$$
\begin{aligned}
& |+(t ; \omega)\rangle=\left(\begin{array}{c}
\sin \frac{\theta}{2} e^{i(\omega / 2) t} \\
\cos \frac{\theta}{2} e^{-i \phi} e^{-i(\omega / 2) t}
\end{array}\right), \\
& |-(t ; \omega)\rangle=\left(\begin{array}{c}
\cos \frac{\theta}{2} e^{i(\omega / 2) t} \\
\sin \frac{\theta}{2} e^{-i \phi} e^{-i(\omega / 2) t}
\end{array}\right), \\
& \epsilon_{ \pm}= \pm \Omega \equiv \pm \sqrt{\Delta^{2}+|V|^{2}}
\end{aligned}
$$

where $\Delta \equiv \Delta_{0}-\hbar \omega / 2$ is the detuning, $\phi=\arg V$ is the coupling phase, $\theta \equiv \arccos (\Delta / \Omega)=\arcsin (|V| / \Omega)$ is the mixing angle, and $\Omega$ is the generalized Rabi frequency.

Consider then driving this system by a frequencymodulated field with time varying $\omega \rightarrow \omega_{t}$. The resulting Hamiltonian can be expressed as a modulation of the form discussed above with $\hat{\widetilde{H}}(t)=\hat{H}_{0}\left(t ; \omega_{t}\right)$, that sweeps through Hamiltonians in the family described by Eq. (17). In general, this problem does not admit a closed-form solution, but is tractable through direct numerical propagation of the TDSE. However, in the limit where the modulation changes sufficiently slowly the dynamics can be solved using the generalized adiabatic theorem for isolated systems [Eq. (7)].

To define the domain in which this theorem applies consider the modulation derivative,

$$
\frac{\partial}{\partial \omega} \tilde{H}(t ; \omega)=\left(\begin{array}{cc}
0 & i t V e^{i \omega t} \\
-i t V^{*} e^{-i \omega t} & 0
\end{array}\right),
$$

which characterizes the effect of the modulation on the driving field. Given Eq. (19), we have

$$
|-(t ; \omega)\rangle \frac{\partial}{\partial \omega} \hat{\widetilde{H}}(t ; \omega)\langle+(t ; \omega)|=i \frac{V \Delta}{\Omega} t,
$$

required for the generalized adiabatic theorem.

Substituting Eqs. (21) and (20) into Eq. (7) shows that the generalized adiabatic theorem applies when

$$
\max _{0 \leq t \leq \tau}\left\{\frac{\left|\dot{\omega}_{t}\right||V| \Delta_{t}}{2 \Omega_{t}^{2}} t\right\} \ll \min _{0 \leq t \leq \tau}\left\{2\left|\Omega_{t}\right|\right\}
$$

where we indicate the parameters that change over time upon frequency modulation using the subscript $t$. Here $\left|\Omega_{t}\right|=\sqrt{\Delta_{t}^{2}+|V|^{2}}$.

When Eq. (22) is well satisfied, then a system initialized in one of the generalized adiabats $| \pm\rangle$ will remain in that state at all times. To demonstrate this, we consider a simple linear modulation $\omega_{t}=\omega_{0}+\dot{\omega} t$, where $\dot{\omega}$ is the constant frequency-sweep velocity, and where the field, initially in resonance $\Delta_{t=0}=0$, is swept linearly to $\Delta_{t=\tau}=4 \Delta_{0}$. In Fig. 1, numerically exact dynamics are obtained for a range of sweep velocities through Runge-Kutta integration of the TDSE. These dynamics are then compared to the adiabatic 


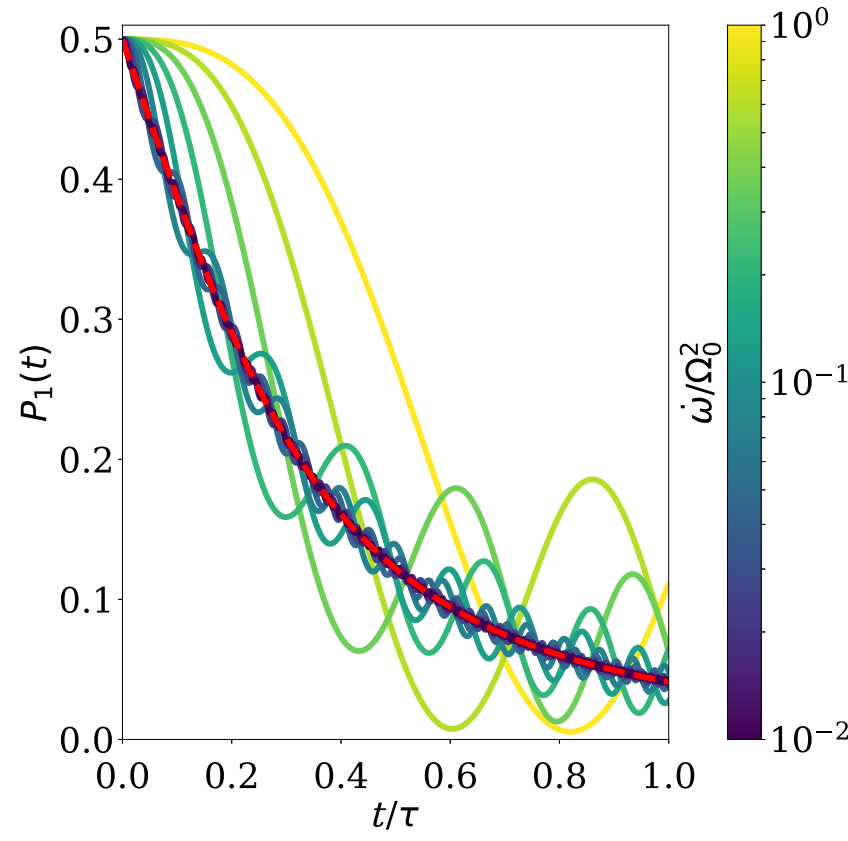

FIG. 1. Dynamics of a two-level system driven by a frequency-modulated field. The frequency of the driving field is swept linearly from a resonant frequency $\hbar \omega_{0}=\Delta_{0}$ to $\hbar \omega_{\tau}=$ $5 \Delta_{0}$ with sweep velocity $\dot{\omega}$ (i.e., $\omega_{t}=\Delta_{0}+\dot{\omega} t$ ). This transformation takes place over a time interval $\tau=4 \Delta_{0} / \dot{\omega}$. The yellow to blue traces show the dynamics under a range of sweep velocities while the red dashed line shows the adiabatic prediction expected for infinitely slow-sweep velocities.

theorem predictions, showing excellent agreement for slow modulations. (We note that the time taken to perform this modulation varies significantly for different sweep velocities, and we plot the dynamics in Fig. 1 on a normalized time axis $t / \tau$.)

This example is helpful in highlighting the construction and flexibility of the AMT. The adiabatic speed limit is not set by the energy gap but rather by the Rabi frequency $\Omega$ on the right-hand side of Eq. (22). This feature, typical of the AMT, is remarkable since it allows for manipulation of the adiabatic time scales simply by increasing the intensity of the driving field $V$, and hence $\left|\Omega_{t}\right|$, and allows for adiabatic transformations of near degenerate systems on manageable timescales. Moreover, the normal modes, given by the Floquet states $| \pm\rangle$, follow the dynamics of the system under fixed frequency driving. By following these oscillatory dynamics the oscillations of the driving field that typically lead to the failure of traditional ATs are removed from consideration [13-16]. In this particularly simple case where only one frequency of light drives the system, this is equivalent to moving to a rotating reference frame using the interaction picture. The instantaneous normal modes can therefore be thought of as a more general method for following the dynamics of the system when an interacting reference frame cannot be defined.

\section{Generalizing adiabatic experiments}

As an indication of the additional role of the AMT in isolated systems, note that it allows a wide range of experimental applications beyond that of the traditional AT. Currently, experimental adiabatic rapid passage and stimulated Raman adiabatic passage techniques have combined the rotating-wave approximation with a rotating reference frame in order to apply the traditional AT to optically driven systems. While this approach can be effective for treating systems driven by near-resonant monochromatic lasers or transform-limited pulses, it imposes several restrictions on the types of experiments that can be realized. The AMT, however, removes many of these limitations, allowing for driving beyond the rotating-wave approximation, driving of a transition by multichromatic fields, and driving of more simultaneous transitions than is allowed under standard conditions. The removal of these limitations can have significant effects on possible experimental techniques. (For example, a recently derived adiabaticity condition [33] allowed for the use of specifically designed pulse sequences to realize adiabatic transformations in finite time [34].) Moreover, the AMT allows for the adiabatic treatment of new phenomena that lie far outside of the rotating-wave regime, such as Sisyphus cooling, and may address experimental challenges that are intractable using simpler driving protocols, such implementing stimulated hyper-Raman adiabatic passage in the presence of Autler-Townes shifts from spectator states [35,36].

To appreciate the advantages afforded by the AMT, consider issues in the application of the traditional AT to oscillatory Hamiltonians. This has been carried out in the interaction picture by moving to a rotating reference frame where the oscillatory Hamiltonian time dependence can be removed. If this can not be done, then the Marzlin-Sanders inconsistency [16] prevents application of the traditional adiabatic theorem. Hence, applications of the traditional AT are limited to systems where an appropriate choice of basis and reference Hamiltonian can be found to remove all oscillatory time dependence.

Consider then the limits of the transformations possible by an appropriate choice of reference Hamiltonian in the interaction picture. Let $H(t)$ be an arbitrary timedependent Hamiltonian

$$
H(t)=\sum_{i} E_{i}(t)|i\rangle\left\langle i\left|+\sum_{i j} V_{i j}(t)\right| i\right\rangle\langle j|,
$$

where $E_{i}(t)$ are the time-dependent diagonal energies and $V_{i j}(t)$ the off-diagonal couplings.

An interaction picture, such as the commonly used rotating reference frame, is defined by a choice of a reference Hamiltonian $H_{0}$. For simplicity, we can work in the eigenbasis of the reference Hamiltonian to give the representation $H_{0}=\sum_{i} E_{i}^{(0)}|i\rangle\langle i|$. Equation (23) can then be 
rewritten in the interaction picture to give

$$
\begin{aligned}
H_{I}(t) & =e^{i\left[H_{0}(t) / \hbar\right] t}\left[H(t)-H_{0}\right] e^{-i\left[H_{0}(t) / \hbar\right] t} \\
& =\sum_{i}\left[E_{i}(t)-E_{i}^{(0)}\right]|i\rangle\left\langle i\left|+\sum_{i j} V_{i j}(t) e^{-i\left[E_{i j}^{(0)} / \hbar\right] t}\right| i\right\rangle\langle j|,
\end{aligned}
$$

where $E_{i j}^{(0)}=E_{i}^{(0)}-E_{j}^{(0)}$.

Considering the conditions under which oscillatory time dependence can be removed from Eq. (24) provides a set of conditions for when AT-based approaches can be applied. This requires a basis in which the diagonal elements of $H(t)$ have no oscillatory time dependence, defining the eigenbasis of the reference Hamiltonian $H_{0}$. The off-diagonal elements show that the only way to eliminate oscillatory time dependence in $V_{i j}(t)$ is if $V_{i j} \propto e^{i \omega_{i j} t}$ for a frequency $\omega_{i j}=E_{i j}^{(0)} / \hbar$. This condition that prescribes a specific functional form for the time dependence of $V_{i j}$ is very restrictive and suggests that the strategy of removing oscillatory Hamiltonian time dependence will only be effective in specific situations. However, this approach has been successful since the oscillatory functional form $V_{i j} \propto$ $e^{-i \omega_{i j} t}$ is precisely what is seen when modeling optically driven transitions in the rotating-wave approximation.

This condition, however, does limit the application of the traditional adiabatic theorem to specific types of optical excitation. First, the excitation must be well modeled using the rotating-wave approximation. If this is not the case, then the off-diagonal matrix elements would be real valued [e.g., of the form $\left.V_{i j} \propto \sin \left(\omega_{i j} t\right)\right]$ and the interaction picture transformation would leave a residual oscillatory component (e.g., of the form $e^{2 i \omega_{i j} t}$ ). Moreover, it precludes the driving of one transition by more than one frequency of light, preventing the use of multicolor excitation. Finally, for a discrete $N$-dimensional system, the $N(N-1)$ off-diagonal elements impose up to $N(N-1)$ conditions $\hbar \omega_{i j}=E_{i j}^{(0)}$ conditions on the $N$ diagonal elements of $H_{0}$. Beyond two-dimensional spin systems, it is not guaranteed that it will be possible to simultaneously satisfy all of these conditions. As a result, removal of oscillatory time dependence can only be guaranteed when a total of $N$ or fewer transitions are driven by an oscillatory external field unless additional resonance conditions are met. The AMT shares none of these limitations.

\section{B. Open-system adiabatic turn on of incoherent light}

A particularly important case in open-system quantum mechanics involves the adiabatic turn on of incoherent radiation that is incident on a molecule and the role of quantum coherences in biological processes (e.g., photosynthesis and vision). In particular, oscillatory coherences have been observed experimentally in the excitation of biological molecules with pulsed lasers [37-40].
By contrast, we have argued, supported by numerical studies, that natural processes rely upon slowly turned on incoherent light, which leads to steady-state transport $[24,41,42]$ with no oscillating coherences. These two sets of studies differ primarily in the nature of the optical fields' driving dynamics. In the laser experiments, the dynamics are induced by sequences of intense femtosecond laser pulses that prepare specific excited state superpositions at a specific time. In contrast, the steady-state studies treat exciting fields that are stochastic white noises (or equivalently broad thermalized photon baths at approximately $5800 \mathrm{~K}$ ) with slowly increasing intensity that continuously excite statistical mixtures of excited states with stochastic phase. The differences in the resulting excitations lead to different predictions of the role of coherences in natural settings and even in the rate of these photoinduced processes in nature [42]. As shown below, the application of the open-system adiabatic theorem proves that if a system is driven by very-slowly turned-on light, then the only coherences that will be observed are those that survive to the steady state. In particular, the pulsed-laser-generated coherences noted above do not survive and are irrelevant under natural biological conditions.

Specifically, consider a molecular system initially prepared in the absence of a driving light field. In this case, the system is initially in a simple equilibrium steady state (i.e., with vanishing dynamical frequency). As such, before the radiation field is turned on, the system is found in a Jordan block with zero eigenvalue. At $t=0$ an incoherent light field is turned on on a time scale much slower than the dynamics of the molecule, a typical circumstance in light-induced biophysical processes. This corresponds to a modulation in the limit of $\dot{\lambda}_{t} \rightarrow 0$, ensuring adiabaticity [see Eq. (16)] of the underlying dynamics. As a result, at all times, the system is found in a Jordan block with vanishing eigenvalue, that is, in a steady state. This then indicates that the only coherences observed in the slow turn-on limit are those that survive in the steady state, such as those seen in previous theoretical studies [24,43-46]. Crucially, this conclusion does not depend on any particular realization of the incoherent light or on any approximation scheme for the dynamics.

In many cases, consistency with thermodynamics requires the system to have one steady state given by the Gibbs state $\rho \propto \exp \left(-\hat{H} / k_{B} T\right)$, which shows no coherences between nondegenerate energy eigenstates. This result suggests that the nonsteady-state coherent dynamics observed under finite turn-on times are a consequence of the rapid turn on of the incoherent light field. Figure 2 discussed below provides a numerical example of these predictions for a popular generic V-system model [24,25].

The ability to show that no coherent dynamics survive the slow turn-on limit of incoherent light without requiring any calculation highlights the intuitive power of the adiabatic theorems derived in this paper. Notably, this 


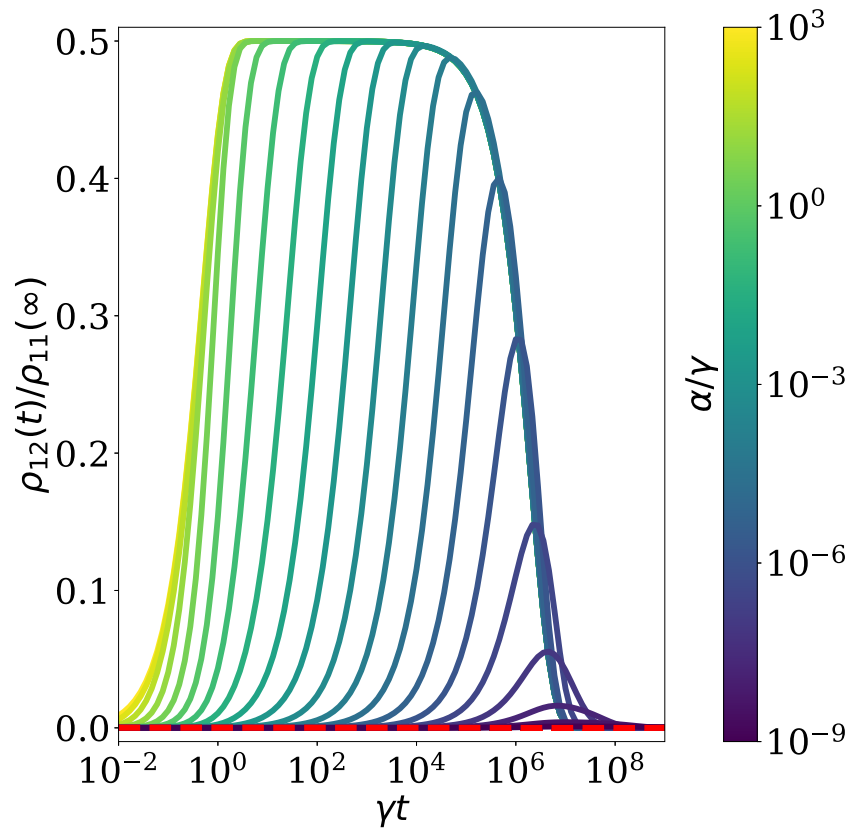

FIG. 2. Dynamics of a $V$ system driven by slowly turned on incoherent light. The intensity of the exciting field, i.e., the mean photon number, turns on with a rate $\alpha$ following an exponential activation function $\bar{n}(t) \propto[1-\exp (-\alpha t)]$. We consider a system driven by a blackbody source with temperature $T=5800 \mathrm{~K}$ with ground to excited state excitation energy of $\omega_{g e}=1.98 \mathrm{eV}$. Both excited states have a natural line width of $\gamma=1 \mathrm{GHz}$ with a splitting of $\Delta=0.001 \hbar \gamma$. The yellow to blue traces show the dynamics under a range of turn-on rates while the dashed red trace shows the adiabatic turn-on prediction.

situation is far beyond the bounds of applicability of previous adiabaticity condition as the system dynamics are driven by a noisy incoherent light field. This produces a driving Hamiltonian that fluctuates extremely rapidly, on the order of $1 \mathrm{fs}$, much faster than the energy gap between states and consequently the underlying molecular dynamics. A numerical example follows below.

Consider then the basic minimal model: an open threelevel system under irradiation by slowly turned-on incoherent light. This model has been previously treated where numerical results showed [25] that coherent dynamics, i.e., coherences in the excited state, vanished under slow turn on of the exciting field. The system has a ground-energy eigenstate, $|g\rangle$, and two excited states, $\left|e_{1}\right\rangle$ and $\left|e_{2}\right\rangle$ that are separated by an energy $\Delta$ and is excited by an incoherent light source with time-dependent intensity. The dynamics of this system, in the weak-coupling limit, is characterized by the following partial secular Bloch-Redfield master equations for the matrix elements of the density operator $\hat{\rho}$ :

$$
\begin{aligned}
\dot{\rho}_{g g}= & -\left[r_{1}(t)+r_{2}(t)\right] \rho_{g g}+\left[r_{1}(t)+\gamma_{1}\right] \rho_{e_{1} e_{1}} \\
& +\left[r_{2}(t)+\gamma_{2}\right] \rho_{e_{2} e_{2}}+2 p\left[\sqrt{r_{1}(t) r_{2}(t)}\right. \\
& \left.+\sqrt{\gamma_{1} \gamma_{2}}\right] \rho_{e_{1} e_{2}}^{R}, \\
\dot{\rho}_{e_{i} e_{i}}= & r_{i}(t) \rho_{g g}-\left[r_{i}(t)+\gamma_{i}\right] \rho_{e_{i} e_{i}} \\
& -p\left[\sqrt{r_{1}(t) r_{2}(t)}+\sqrt{\gamma_{1} \gamma_{2}}\right] \rho_{e_{1} e_{2}}^{R}, \\
\dot{\rho}_{e_{1} e_{2}}= & -\frac{1}{2}\left[r_{1}(t)+r_{2}(t)+\gamma_{1}+\gamma_{2}+2 i \Delta\right] \rho_{e_{1} e_{2}} \\
& +\frac{p}{2} \sqrt{r_{1}(t) r_{2}(t)}\left(2 \rho_{g g}-\rho_{e_{1} e_{1}}-\rho_{e_{2} e_{2}}\right) \\
& -\frac{p}{2} \sqrt{\gamma_{1} \gamma_{2}}\left(\rho_{e_{1} e_{1}}+\rho_{e_{2} e_{2}}\right),
\end{aligned}
$$

where $\gamma_{i}$ is the spontaneous emission rate and $r_{i}(t)$ is the rate of excitation to (and stimulated emission from) state $\left|e_{i}\right\rangle$. The alignment parameter $p$ characterizes the probability of simultaneous excitation to states $\left|e_{1}\right\rangle$ and $\left|e_{2}\right\rangle$ and is defined as

$$
p \equiv \frac{\boldsymbol{\mu}_{\mathbf{1}} \cdot \boldsymbol{\mu}_{\mathbf{2}}}{\mu_{1} \mu_{2}}
$$

where $\mu_{i}$ is the transition dipole moment between the ground state $|g\rangle$ and excited state $\left|e_{i}\right\rangle$, assumed real for simplicity. The spontaneous emission and excitation rates are related by detailed balance to give $r_{i}(t)=\gamma_{i} \bar{n}(t)$ where $\bar{n}(t)$ is the mean occupation number of the resonant thermal field mode. It is the slow turn on of the incoherent light that leads to a time-dependent occupation number $\bar{n}(t)$ of the thermal field reflecting its time-dependent intensity. That is, the slow modulation of the Liouvillian arises due to slow changes in the statistics of the exciting field, in this case in the mean number of photons in the thermal field modes.

In order to apply the open-system AMT, it is useful to write Eq. $(25 \mathrm{c})$ in matrix form. By writing $|\rho\rangle\rangle=$ $\left[\rho_{g g}, \rho_{e_{1} e_{1}}, \rho_{e_{2} e_{2}}, \rho_{e_{1} e_{2}}, \rho_{e_{2} e_{1}}\right]^{T}$ as a column vector of the density-matrix elements, Eq. (25c) can be written in the $\mathrm{LvN}$ form of Eq. (8), where $\hat{\hat{\mathcal{L}}}(t)$ is a matrix given by

$$
\hat{\hat{\mathcal{L}}}(t)=\left[\begin{array}{ccccc}
-\left[r_{1}(t)+r_{2}(t)\right] & r_{1}(t)+\gamma_{1} & r_{2}(t)+\gamma_{2} & 2 p\left[\sqrt{r_{1}(t) r_{2}(t)}+\sqrt{\gamma_{1} \gamma_{2}}\right] & 2 p\left[r_{12}(t)+\gamma_{12}\right] \\
r_{1}(t) & -\left[r_{1}(t)+\gamma_{1}\right] & 0 & -\frac{p}{2}\left[r_{12}(t)+\gamma_{12}\right] & -\frac{p}{2}\left[r_{12}(t)+\gamma_{12}\right] \\
r_{2}(t) & 0 & -\left[r_{2}(t)+\gamma_{2}\right] & -\frac{p}{2}\left[r_{12}(t)+\gamma_{12}\right] & -\frac{p}{2}\left[r_{12}(t)+\gamma_{12}\right] \\
p r_{12}(t) & -\frac{p}{2}\left[r_{12}(t)+\gamma_{12}\right] & -\frac{p}{2}\left[r_{12}(t)+\gamma_{12}\right] & -i \Delta-\bar{r}-\bar{\gamma} & 0 \\
p r_{12}(t) & -\frac{p}{2}\left[r_{12}(t)+\gamma_{12}\right] & -\frac{p}{2}\left[r_{12}(t)+\gamma_{12}\right] & -i \Delta-\bar{r}-\bar{\gamma} & 0
\end{array}\right],
$$


where $r_{12}(t)=\sqrt{r_{1}(t) r_{2}(2)}$ and $\gamma_{12}=\sqrt{\gamma_{1} \gamma_{2}}$ are the geometric mean pumping and decay rates and $\bar{r}(t)=\left[r_{1}(t)+\right.$ $\left.r_{2}(t)\right] / 2$ and $\bar{\gamma}=\left(\gamma_{1}+\gamma_{2}\right) / 2$ are the corresponding arithmetic means. Equation (27) is analogous to writing the Hamiltonian as a matrix when expressing the TDSE in matrix form with the exception that the Liouvillian is not Hermitian. In this form, the open-system AMT is applied analogously to the isolated-system AMT as was done in Sec. III A1, using the Liouvillian in place of the Hamiltonian. The only added complication that can arise is that the operator may not be diagonalizable since it is not Hermitian, in which case it must be placed into Jordan normal form using standard linear algebra techniques [47]. In this section, we pursue an alternative route, since we are interested in the simpler question of estimating the dynamics in the limit of an infinitely slow turn-on time. This requires only evaluating the steady state of the Liouvillian (i.e., the $\chi_{\alpha}=0$ ) eigenspace at various fixed values of $r_{i}$, since the system is initialized in the ground state - the steady state at the initial $r_{i}(0)=0$ field.

These equations of motion can be analytically solved by diagonalizing the matrix in Eq. (27) giving several distinct dynamical regimes [25]. In particular, in the limit where $\bar{\gamma} / \Delta_{P} \gg 1$, with $\Delta_{p}=\sqrt{\Delta^{2}+\left(1-p^{2}\right) \gamma_{1} \gamma_{2}}$, a regime examined below, the system shows long-lived quasistationary coherences that eventually decay to give an incoherent thermal state [48]. Under excitation by a field with time-dependent intensity of the form $\bar{n}(t)=$ $\bar{n}[1-\exp (-\alpha t)]$, the dynamics are given by

$$
\begin{aligned}
& \rho_{e_{i} e_{i}}(t)=\frac{1}{2 \bar{\gamma}}\left\{\frac{r_{j}}{\left(\Delta_{p}^{2} / 2 \bar{\gamma}\right)-\alpha}\left[\frac{\Delta_{p}^{2}}{2 \bar{\gamma}}\left(1-e^{-\alpha t}\right)-\alpha\left(1-e^{-\frac{\Delta_{p}^{2}}{2 \bar{\gamma}} t}\right)\right]+\frac{r_{i}}{2 \bar{\gamma}-\alpha}\left[2 \bar{\gamma}\left(1-e^{-\alpha t}\right)-\alpha\left(1-e^{-2 \bar{\gamma} t}\right)\right]\right\} \quad(28 \mathrm{a}) \\
& \rho_{e_{1} e_{2}}(t)=\frac{p \sqrt{r_{1} r_{2}}}{2 \bar{\gamma}}\left\{\frac{1}{\left(\Delta_{p}^{2} / 2 \bar{\gamma}\right)-\alpha}\left[\frac{\Delta_{p}^{2}}{2 \bar{\gamma}}\left(1-e^{-\alpha t}\right)-\alpha\left(1-e^{-\frac{\Delta_{p}^{2}}{2 \bar{\gamma}} t}\right)\right]+\frac{1}{2 \bar{\gamma}-\alpha}\left[2 \bar{\gamma}\left(1-e^{-\alpha t}\right)-\alpha\left(1-e^{-2 \bar{\gamma} t}\right)\right]\right\},
\end{aligned}
$$

with $\bar{\gamma}$ and $\Delta_{p}$ defined above. A detailed derivation of Eq. (28) along with an in-depth discussion of the underlying physics are given in Ref. [25].

While Eq. (25) is complicated, the dynamics are qualitatively simple. When the turn-on time $\tau_{r}=\alpha^{-1}$ is shorter than the coherence lifetime $2 \bar{\gamma} / \Delta_{p}^{2}$, the quasistationary coherences lead to a potentially long-lived superposition of excited states, which rises on a timescale $\tau_{r}$. The coherences then decay on a timescale $2 \bar{\gamma} / \Delta_{p}^{2}$. As the turn-on time becomes comparable to or longer than the coherence lifetime, the quasistationary superposition is not fully excited, leading to a decrease in the generated coherences that disappear entirely in the $\tau_{r} \rightarrow \infty$ limit. These dynamics are shown for a variety of turn-on rates, $\alpha$, in the solid traces of Fig. 2 for the case of $p=1, \gamma_{1}=\gamma_{2}$.

These traces can be compared to the dashed red trace, which shows the predictions of the open-system adiabatic theorem. As discussed above, since the system is initialized in a steady state of the zero-field case, a sufficiently slow turn on leads to dynamics that remain in the instantaneous steady state at $\bar{n}(t)$. In this example involving only a single bath, the steady state is the thermal Gibbs state with a temperature determined by $\bar{n}(t)$, and therefore shows no coherences. Consequently, as illustrated in this example, the open-system adiabatic theorem guarantees that a sufficiently slow turn on of an incoherent field will only show coherences that survive in the steady state. In the case of two baths, steady-state coherences associated with transport will persist $[24,43]$.

\section{CONCLUSION}

We present a new set of generalized adiabatic theorems that apply to rapidly fluctuating fields with slowly varying properties. These results express the dynamics of the modulated field in terms of the dynamical normal modes of time-dependent reference Hamiltonians. The resulting adiabaticity conditions take a form that is conceptually similar to pre-existing theorems but bounds adiabaticity based on the rate of a slowly changing transformation rather than the rapid underlying field. These results significantly extend the applicability of the adiabatic theorem and its underlying intuition to, e.g., the domain of optical excitations that play a central role in atomic, molecular, and optical physics.

The AMTs presented in this work provide fundamental insights into quantum dynamics and can be applied to develop new techniques in quantum control, sensing, and information processing.

Fundamentally, these theorems show that the notions of adiabaticity first formulated for eigenstates of slowly varying Hamiltonians can be extended to fields that oscillate 
on arbitrary timescales similar to or even faster than the system dynamics. As illustrated by the example of incoherent excitation of biomolecular systems in Sec. III B, these concepts can significantly simplify our understanding of highly complex dynamical problems involving rapidly oscillating and stochastic fields by revealing that, in some cases, their behavior is primarily determined by a much simpler set of slowly varying properties. These generalized adiabaticity concepts can enable the use of much simpler models or numerical techniques to describe previously intractable or challenging problems in these regimes, similarly to the application of the adiabatic theorem to justify existing adiabatic quantum dynamics method. As a concrete example, we show that incoherent excitation by adiabatically turned-on light can be understood by simply calculating the steady states at different intensities rather than undertaking the generally far more complicated task of full dynamic propagation.

Practically, the AMTs significantly expand the design space of quantum adiabatic transformations such as ARP and STIRAP by enabling the use of rapidly varying timedependent fields, which can allow for the design of protocols that are faster and more robust to environmental noise and system heterogeneity (e.g., by exploiting combinations of resonant fields to induce rapid dynamics). These methods have found wide-spread applicability in a variety of fields due to their ability to robustly transform one quantum state into another, including in adiabatic [7] and circuit-based quantum computing [49-52], gasphase spectroscopy $[19,20]$, single-atom cavity quantum electrodynamics [53-57] and the preparation of ultracold molecules [58-61]. The ability to accelerate adiabatic protocols can present important new opportunities in these fields as the success of adiabatic transformations in realistic settings is often limited by their ability to out-race external relaxation channels.

In addition to accelerating adiabatic protocols in existing applications, the AMTs allow for a fundamentally new type of adiabatic transformation. While previously reported ATs are restricted to transformations from one static eigenstate to another, the AMTs allow for transformations from one type of dynamics to another. For example, given a three-level quantum system with states $|0\rangle,|1\rangle$, and $|2\rangle$, previous ATs could realize transformations from one of these states to another (e.g., from $|0\rangle$ to |1)). The AMTs also allow for transformations that start with a wave function that dynamically cycles between the states in one direction (e.g., $|0\rangle \rightarrow|1\rangle \rightarrow|2\rangle$ ) and ends with a wave function that cycles in the opposite direction $(|0\rangle \rightarrow|2\rangle \rightarrow|1\rangle)$. This new ability to control dynamics and not just state preparation may open new applications for adiabatic control protocols that would not be possible with previous results (e.g., in quantum thermodynamics where controlling the direction of flux around a cycle may be useful).

\section{ACKNOWLEDGMENTS}

This work is supported by the US Air Force Office of Scientific Research Under Contracts No. FA9550-17-10310 and No. FA9550-20-1-0354.

[1] M. Born and V. Fock, Beweis des adiabatensatzes, Z. Phys. 51, 165 (1928).

[2] T. Kato, On the adiabatic theorem of quantum Mechanics, J. Phys. Soc. Jpn. 5, 435 (1950).

[3] M. S. Sarandy and D. A. Lidar, Adiabatic approximation in open quantum systems, Phys. Rev. A 71, 012331 (2005).

[4] A. Messiah, Quantum Mechanics: Two Volumes Bound as One (Dover Publications, Mineola, NY, 2017).

[5] L. D. Landau, Zur theorie der energieubertragung. ii. Phys. Z. Sowjetunion 2, 1 (1932).

[6] C. Zener, Non-adiabatic crossing of energy levels, Proc. R. Soc. London A 137, 696 (1932).

[7] T. Albash and D. A. Lidar, Adiabatic quantum computation, Rev. Mod. Phys. 90, 015002 (2018).

[8] R. Barends, et al., Digitized adiabatic quantum computing with a superconducting circuit, Nature 534, 222 (2016).

[9] E. Torrontegui, I. Lizuain, S. González-Resines, A. Tobalina, A. Ruschhaupt, R. Kosloff, and J. G. Muga, Energy consumption for shortcuts to adiabaticity, Phys. Rev. A 96, 022133 (2017).

[10] A. del Campo, Shortcuts to Adiabaticity by Counterdiabatic Driving, Phys. Rev. Lett. 111, 100502 (2013).

[11] E. Torrontegui, S. Ibáñez, S. Martínez-Garaot, M. Modugno, A. del Campo, D. Guéry-Odelin, A. Ruschhaupt, X. Chen, and J. G. Muga, in Advances in Atomic, Molecular, and Optical Physics, edited by E. Arimondo, P. R. Berman, and C. C. Lin (Academic Press, San Diego, California, USA, 2013), Vol. 62, p. 117.

[12] A. D. Campo and M. G. Boshier, Shortcuts to adiabaticity in a time-dependent box, Sci. Rep. 2, 648 (2012).

[13] M. H. S. Amin, Consistency of the Adiabatic Theorem, Phys. Rev. Lett. 102, 220401 (2009).

[14] J. Du, L. Hu, Y. Wang, J. Wu, M. Zhao, and D. Suter, Experimental Study of the Validity of Quantitative Conditions in the Quantum Adiabatic Theorem, Phys. Rev. Lett. 101, 060403 (2008).

[15] D. M. Tong, K. Singh, L. C. Kwek, and C. H. Oh, Quantitative Conditions Do Not Guarantee the Validity of the Adiabatic Approximation, Phys. Rev. Lett. 95, 110407 (2005).

[16] K.-P. Marzlin and B. C. Sanders, Inconsistency in the Application of the Adiabatic Theorem, Phys. Rev. Lett. 93, 160408 (2004).

[17] R. Dann and R. Kosloff, Inertial theorem: Overcoming the quantum adiabatic limit, Phys. Rev. Res. 3, 013064 (2021).

[18] V. S. Malinovsky and J. L. Krause, General theory of population transfer by adiabatic rapid passage with intense, chirped laser pulses, Eur. Phys. J. D 14, 147 (2001).

[19] N. V. Vitanov, A. A. Rangelov, B. W. Shore, and K. Bergmann, Stimulated raman adiabatic passage in physics, chemistry, and beyond, Rev. Mod. Phys. 89, 015006 (2017). 
[20] K. Bergmann, N. V. Vitanov, and B. W. Shore, Perspective: Stimulated raman adiabatic passage: The status after 25 years, J. Chem. Phys. 142, 170901 (2015).

[21] O. Kaufmann, A. Ekers, C. Gebauer-Rochholz, K. U. Mettendorf, M. Keil, and K. Bergmann, Dissociative charge transfer from highly excited $\mathrm{Na}$ Rydberg atoms to vibrationally excited $\mathrm{Na}_{2}$ molecules, Int. J. Mass Spectrom. Low Energy Electron-Mol. Interact. (Stamatovic Honor) 205, 233 (2001).

[22] M. Külz, M. Keil, A. Kortyna, B. Schellhaaß, J. Hauck, K. Bergmann, W. Meyer, and D. Weyh, Dissociative attachment of low-energy electrons to state-selected diatomic molecules, Phys. Rev. A 53, 3324 (1996).

[23] K. Aikawa, D. Akamatsu, M. Hayashi, K. Oasa, J. Kobayashi, P. Naidon, T. Kishimoto, M. Ueda, and S. Inouye, Coherent Transfer of Photoassociated Molecules Into the Rovibrational Ground State, Phys. Rev. Lett. 105, 203001 (2010).

[24] A. Dodin and P. Brumer, Light-induced processes in nature: Coherences in the establishment of the nonequilibrium steady state in model retinal isomerization, J. Chem. Phys. 150, 184304 (2019).

[25] A. Dodin, T. V. Tscherbul, and P. Brumer, Coherent dynamics of V-type systems driven by time-dependent incoherent radiation, J. Chem. Phys. 145, 244313 (2016).

[26] Y.-C. Cheng and G. R. Fleming, Dynamics of light harvesting in photosynthesis, Annu. Rev. Phys. Chem. 60, 241 (2009).

[27] A. Chenu and G. D. Scholes, Coherence in energy transfer and photosynthesis, Annu. Rev. Phys. Chem. 66, 69 (2015).

[28] C. L. Byrne, Signal Processing: A Mathematical Approach (A K Peters/CRC Press, Wellesley, Mass, 1993).

[29] The modified Hamiltonian is defined using a partial time derivative and therefore neglects the implicit time dependence of $\lambda_{t}$.

[30] H.-P. Breuer and F. Petruccione, The Theory of Open Quantum Systems (Oxford University Press, Oxford, United Kingdom, 2007).

[31] R. Alicki and K. Lendi, Quantum Dynamical Semigroups and Applications in Lecture Notes in Physics, Vol. 286 (Springer-Verlag Berlin Heidelberg, Berlin, Germany, 2007).

[32] K. Blum, Density Matrix Theory and Applications (Springer Science \& Business Media, New York, USA, 2012).

[33] Z.-Y. Wang and M. B. Plenio, Necessary and sufficient condition for quantum adiabatic evolution by unitary control fields, Phys. Rev. A 93, 052107 (2016).

[34] K. Xu, T. Xie, F. Shi, Z.-Y. Wang, X. Xu, P. Wang, Y. Wang, M. B. Plenio, and J. Du, Breaking the quantum adiabatic speed limit by jumping along geodesics, Sci. Adv. 5, eaax3800 (2019).

[35] S. Guérin, L. P. Yatsenko, T. Halfmann, B. W. Shore, and K. Bergmann, Stimulated hyper-raman adiabatic passage. II. Static compensation of dynamic stark shifts, Phys. Rev. A 58, 4691 (1998).

[36] L. P. Yatsenko, S. Guérin, T. Halfmann, K. Böhmer, B. W. Shore, and K. Bergmann, Stimulated hyper-raman adiabatic passage. I. The basic problem and examples, Phys. Rev. A 58, 4683 (1998).
[37] G. S. Engel, T. R. Calhoun, E. L. Read, T.-K. Ahn, T. Mancal, Y.-C. Cheng, R. E. Blankenship, and G. R. Fleming, Evidence for wavelike energy transfer through quantum coherence in photosynthetic systems, Nature (London) 446, 782 (2007).

[38] Y. Zhang, S. Oh, F. H. Alharbi, G. S. Engel, and S. Kais, Delocalized quantum states enhance photocell efficiency, Phys. Chem. Chem. Phys. 17, 5743 (2015).

[39] A. Ishizaki, T. R. Calhoun, G. S. Schlau-Cohen, and G. R. Fleming, Quantum coherence and its interplay with protein environments in photosynthetic electronic energy transfer, Phys. Chem. Chem. Phys. 12, 7319 (2010).

[40] P. J. M. Johnson, A. Halpin, T. Morizumi, V. I. Prokhorenko, O. P. Ernst, and R. J. D. Miller, Local vibrational coherences drive the primary photochemistry of vision, Nat. Chem. 7, 980 (2015).

[41] X.-P. Jiang and P. Brumer, Creation and dynamics of molecular states prepared with coherent vs partially coherent pulsed light, J. Chem. Phys. 94, 5833 (1991).

[42] P. Brumer, Shedding (incoherent) light on quantum effects in light-induced biological processes, J. Phys. Chem. Lett. 9, 2946 (2018).

[43] S. Koyu, A. Dodin, P. Brumer, and T. V. Tscherbul, ArXiv:2001.09230 (2020).

[44] M. Reppert, D. Reppert, L. A. Pachon, and P. Brumer, ArXiv:1911.07606 (2019).

[45] S. Axelrod and P. Brumer, An efficient approach to the quantum dynamics and rates of processes induced by natural incoherent light, J. Chem. Phys. 149, 114104 (2018).

[46] S. Axelrod and P. Brumer, Multiple time scale open systems: Reaction rates and quantum coherence in model retinal photoisomerization under incoherent excitation. J. Chem. Phys. OSQD2019, 014104 (2019).

[47] D. B. Damiano and J. B. Little, A Course in Linear Algebra (Dover Publications, Mineola, NY, 2011), Illustrated edition ed.

[48] T. V. Tscherbul and P. Brumer, Long-Lived Quasistationary Coherences in a V-type System Driven by Incoherent Light. Phys. Rev. Lett. 113, 113601 (2014).

[49] D. Møller, J. L. Sørensen, J. B. Thomsen, and M. Drewsen, Efficient qubit detection using alkaline-earth-metal ions and a double stimulated raman adiabatic process, Phys. Rev. A 76, 062321 (2007).

[50] F. Vewinger, J. Appel, E. Figueroa, and A. I. Lvovsky, Adiabatic frequency conversion of optical information in atomic vapor, Opt. Lett., OL 32, 2771 (2007).

[51] S. C. Webster, S. Weidt, K. Lake, J. J. McLoughlin, and W. K. Hensinger, Simple Manipulation of a Microwave Dressed-State Ion Qubit, Phys. Rev. Lett. 111, 140501 (2013).

[52] N. Timoney, I. Baumgart, M. Johanning, A. F. Varón, M. B. Plenio, A. Retzker, and C. Wunderlich, Quantum gates and memory using microwave-dressed states, Nature 476, 185 (2011).

[53] A. S. Parkins, P. Marte, P. Zoller, and H. J. Kimble, Synthesis of Arbitrary Quantum States via Adiabatic Transfer of Zeeman Coherence, Phys. Rev. Lett. 71, 3095 (1993).

[54] M. Hennrich, T. Legero, A. Kuhn, and G. Rempe, VacuumStimulated Raman Scattering Based on Adiabatic Passage 
in a High-Finesse Optical Cavity, Phys. Rev. Lett. 85, 4872 (2000).

[55] C. Nölleke, A. Neuzner, A. Reiserer, C. Hahn, G. Rempe, and S. Ritter, Efficient Teleportation Between Remote Single-Atom Quantum Memories, Phys. Rev. Lett. 110, 140403 (2013).

[56] T. Wilk, S. C. Webster, A. Kuhn, and G. Rempe, Singleatom single-photon quantum interface, Science 317, 488 (2007).

[57] T. Wilk, S. C. Webster, H. P. Specht, G. Rempe, and A. Kuhn, Polarization-Controlled Single Photons, Phys. Rev. Lett. 98, 063601 (2007).

[58] K. Winkler, F. Lang, G. Thalhammer, P. V. D. Straten, R. Grimm, and J. H. Denschlag, Coherent Optical Transfer of
Feshbach Molecules to a Lower Vibrational State, Phys. Rev. Lett. 98, 043201 (2007).

[59] J. G. Danzl, E. Haller, M. Gustavsson, M. J. Mark, R. Hart, N. Bouloufa, O. Dulieu, H. Ritsch, and H.-C. Nägerl, Quantum gas of deeply bound ground state molecules, Science 321, 1062 (2008).

[60] F. Lang, K. Winkler, C. Strauss, R. Grimm, and J. H. Denschlag, Ultracold Triplet Molecules in the Rovibrational Ground State, Phys. Rev. Lett. 101, 133005 (2008).

[61] K.-K. Ni, S. Ospelkaus, M. H. G. D. Miranda, A. Pe'er, B. Neyenhuis, J. J. Zirbel, S. Kotochigova, P. S. Julienne, D. S. Jin, and J. Ye, A high phase-space-density gas of polar molecules, Science 322, 231 (2008). 